\title{
Las brechas socioeconómicas y su relación con la corrupción: elementos clave a la hora de formular políticas públicas ${ }^{*}$
}

\author{
Leyner Mosquera* \\ Alexandra González Zapata
}

Recibido: 10 de agosto de 2019 • Aprobado: 18 de noviembre de 2019

\section{Resumen}

En el presente documento, se abordan elementos que aportan evidencia empírica para la construcción y puesta en marcha de políticas públicas eficientes, efectivas y eficaces, acorde a las características regionales de Colombia. Se analiza de forma descriptiva el panorama de las políticas públicas en Colombia y se exponen las dinámicas del contexto socioeconómico de Colombia a nivel de subregiones, mediante un estudio de caso sobre el litoral Pacífico. Finalmente, se desarrolla un análisis empírico con un modelo de lineales con efectos fijos y se aplica la metodología de panel. Los principales resultados sugieren que la desigualdad, las brechas sociales

* El título del proyecto de investigación al cual se adscribe el presente artículo es "Observatorio de Política Pública de Control Fiscal de la Auditoría General de la República", gestionado en la Auditoría General de la República de Colombia.

Citar como: Mosquera, L. y González Zapata, A. (2020). Las brechas socioeconómicas y su relación con la corrupción: elementos clave a la hora de formular políticas públicas. Revista IUSTA, 52, 103-122. DoI: https://doi.org/10.15332/25005286.5485

** Ingeniero de Producción Industrial, Máster en Economía Aplicada de la Universidad de los Andes. Asesor del despacho de la Dirección Nacional de la Escuela Superior de Administración Pública. Investigador del Semillero Pacífico de la Facultad de Economía de la Universidad de los Andes. Investigador del "Observatorio de Política Pública del Control Fiscal" adscrito -según convenio-a la Auditoría General de la República.1.mosquera@uniandes.edu.co. orcid: 0000-0001-8431-3482.

**** Politóloga, Máster en Políticas Públicas de la Universidad Nacional de Colombia. Docente en el área de investigación del Departamento de Derecho de la misma universidad. Experiencia en análisis de política pública, política criminal y derechos humanos. Investigadora del Observatorio de Política Pública del Control de la Auditoría General de la República.apgonzalezz@unal.edu.co. ORCID: 0000-0002-6016-7123. 
y económicas están correlacionadas con la corrupción. El presente trabajo se une a la amplia literatura de elementos para diseñar políticas públicas de mayor impacto en países con amplia desigualdad, como es el caso de Colombia.

Palabras clave: Corrupción. Costa pacífica. Inequidad. Políticas públicas.

\title{
SOCIO-ECONOMIC GAPS AND THEIR RELATIONSHIP WITH CORRUPTION: KEY ELEMENTS WHEN FORMULATING PUBLIC POLICIES
}

\begin{abstract}
This document addresses elements that provide empirical evidence for the construction and implementation of efficient, effective and operative public policies, according to the regional characteristics of Colombia. The public policy overview in Colombia is descriptively analyzed and the dynamics of Colombia's socioeconomic context at sub-region level are presented, through a case study on the Pacific coast. Finally, an empirical analysis is developed with a linear model with fixed effects and the panel methodology is applied. The main results suggest that inequality, social and economic gaps are correlated with corruption. This paper joins the extensive literature of elements to design public policies of greater impact in countries with wide inequality, as is the case in Colombia.
\end{abstract}

Keywords: Corruption, Pacific coast, inequality, public policies.

\section{As BRECHAS SOCIOECONÔMICAS E SUA RELAÇÃO COM A CORRUPÇÃO: ELEMENTOS CHAVES NO MOMENTO DE FORMULAR POLÍTICAS PÚBLICAS}

\section{Resumo}

Neste documento abordam-se elementos que aportam evidencia empírica para a construção e implementação de políticas públicas eficientes, efetivas e eficazes, acorde com as características regionais da Colômbia. Analisa-se de forma descritiva o panorama das políticas públicas na Colômbia e expõem-se as dinâmicas do contexto socioeconômico da Colômbia no nível de sub-regiões, mediante um estudo de caso sobre o litoral Pacífico. Finalmente, desenvolve-se uma análise empírica 
com um modelo de lineais com efeitos fixos e aplica-se a metodologia de painel. Os principais resultados sugerem que a desigualdade, a brechas sociais e econômicas estão correlacionada com a corrupção. Este trabalho se une à ampla literatura de elementos para propor políticas públicas de maior impacto em países com ampla desigualdade como é o caso da Colômbia.

Palavras-chave: corrupção, litoral pacífico, iniquidade, políticas públicas.

\section{Introducción}

Colombia es un país heterogéneo, en términos de diversidad cultural, económica, social y de costumbres (Sánchez, 2012). Es muy importante tener esto presente cuando se proponen políticas públicas que apuntan a cerrar brechas socioeconómicas. Es importante comprender que incluso hay diversidad en una misma región. En el caso del Pacífico colombiano, entre el Pacífico andino ${ }^{1}$ y el litoral $\mathrm{Pacífico}^{2}$ existe una gran diversidad no solo en términos culturales, sino también de condiciones socioeconómicas y desigualdad. A pesar de ser la misma región, existen brechas muy amplias con respecto a los indicadores socioeconómicos (Cárdenas, 2018). Un buen ejemplo es Buenaventura: solo está ubicada a dos horas y media de viaje desde la ciudad de Cali, en el departamento del Valle, y las brechas entre ambas ciudades son abismales. De acuerdo a lo anterior, se analizará mediante un estudio de caso las dinámicas socioeconómicas del litoral Pacífico, para entender la realidad del país en las regiones, y la importancia de tener estos aspectos en cuenta a la hora de estructurar políticas públicas (Blanco, 2015).

Otro aspecto importante es analizar las políticas públicas teniendo en cuenta los niveles de corrupción de una región, y su relación con la desigualdad y el

1 Estos son los municipios y ciudades principales de los departamentos de Valle, Cauca y Nariño, ubicados hacia la región Andina del Pacífico colombiano.

2 El litoral Pacífico está conformado por los municipios ubicados en el extremo occidental de la región Pacífica, comprende la franja costera del Pacífico y todos los municipios del Chocó. Estos son los territorios más periféricos, integrados mayoritariamente por territorios colectivos de comunidades negras, resguardos indígenas y áreas protegidas. En el norte, se encuentra el departamento del Chocó, y sus 30 municipios coloreado con un tono azul claro; seguido de Buenaventura, con el tono azul más oscuro; luego el litoral caucano, compuesto por los municipios: Balboa, Buenos Aires, Caldono, Caloto, Corinto, El Tambo, Guachené, Guapi, Jambaló, López, Miranda, Morales, Padilla, Puerto Tejada, Santander de Quilichao, Suárez, Timbiquí, Toribio y Villa Rica. Finalmente, se encuentra el litoral nariñense, compuesto por los municipios: Barbacoas, El Charco, La Tola, Magüi, Mosquera, Olaya Herrera, Francisco Pizarro, Roberto Payán, Santa Bárbara, San Andrés de Tumaco. 
ingreso per cápita. Para esto, se analizan datos de los hallazgos de las contralorías territoriales en los procedimientos de vigilancia fiscal, con el propósito de analizar la correlación entre estos hallazgos y el desempeño fiscal de los departamentos. Estos aspectos podrían jugar un papel importante en este fenómeno. Procesar, analizar y comprender los datos disponibles de corrupción, datos socioeconómicos, de desigualdad (coeficiente Gini), demográficos y de desempeño fiscal, es vital para aportar evidencia empírica a la discusión y considerar de forma integral otros aspectos sociales y económicos que podrían estar jugando un papel preponderante, para la formulación de políticas públicas en Colombia.

En el presente estudio, primero se realiza un breve contexto de las políticas públicas en Colombia, luego se aborda un análisis de estudio de caso en la región del Pacífico colombiano, después se plantea la metodología y descripción de los datos, y finalmente se plantean los resultados y las conclusiones.

\section{Contexto de las políticas públicas en Colombia}

Las políticas públicas se refieren a la forma en que se definen y contribuyen cuestiones y problemas, y a la forma en que llegan a la agenda política y a la agenda de las políticas públicas. A su vez, estudian: “cómo, por qué y para qué los gobiernos adoptan y actúan o no actúan" (Parsons, 1995, p. 248).

En el diseño e implementación de una política pública que atienda las necesidades de la población, el gobierno establece una relación horizontal con la sociedad civil (Benavente, 2014), para lo cual es fundamental contar con la participación activa de los ciudadanos. La Procuraduría General de la Nación señala que:

La política pública es el resultado de un proceso social, no de un mandato jurídico ni de una decisión administrativa, es, por el contrario, la concreción de unos ideales que se construyen a partir de unas dinámicas sociales que requieren ser interpretadas y llevadas a una toma de decisiones capaces de ser administradas y continuamente revisadas. (Procuraduría General de la Nación, 2011)

Por otra parte, la Constitución política de Colombia, en los artículos 1, 2 y 3, hace referencia a la política pública de la siguiente manera: 
Artículo 1. Colombia es un Estado social de derecho, organizado en forma de República unitaria, descentralizada, con autonomía de sus entidades territoriales, democrática, participativa y pluralista, fundada en el respeto de la dignidad humana, en el trabajo y la solidaridad de las personas que la integran y en la prevalencia del interés general.

Artículo 2. Son fines esenciales del Estado: servir a la comunidad, promover la prosperidad general y garantizar la efectividad de los principios, derechos y deberes consagrados en la Constitución; facilitar la participación de todos en las decisiones que los afectan y en la vida económica, política, administrativa y cultural de la Nación; defender la independencia nacional, mantener la integridad territorial y asegurar la convivencia pacífica y la vigencia de un orden justo.

Las autoridades de la República están instituidas para proteger a todas las personas residentes en Colombia, en su vida, honra, bienes, creencias, y demás derechos y libertades, y para asegurar el cumplimiento de los deberes sociales del Estado y de los particulares.

Artículo 3. La soberanía reside exclusivamente en el pueblo, del cual emana el poder público. El pueblo la ejerce en forma directa o por medio de sus representantes, en los términos que la Constitución establece. (Const., 1991)

De acuerdo con lo anterior, se puede establecer que la política pública exhibe un programa concreto que asocia estrategias, decisiones, acciones y evaluaciones, sobre la base de unos indicadores apropiados al seguimiento de una o varias situaciones que deben impactarse, en consideración a unos objetivos claramente establecidos (Torres, 2006).

De acuerdo con la visión de la Alcaldía Distrital de Bogotá, la política pública se define como:

[...] una visión de largo plazo que sobrepasa los periodos de administración de los gobiernos y orienta el proceso de cambio frente a realidades sociales relevantes. Es un instrumento de planeación orientado a la acción para lograr objetivos prioritarios, fruto de un proceso de concertación intersectorial y co-creación, en el que participa la administración distrital, la ciudadanía, la sociedad civil, los gremios, la academia, etc. 
Este proyecto colectivo, como instrumento que favorece la cooperación entre diferentes sectores de la sociedad, exige un trabajo intersectorial por parte de las entidades del Gobierno Distrital y de estas con la ciudadanía, para establecer de forma conjunta la manera de abordar las necesidades más importantes de la ciudad, los enfoques de derechos humanos, género, poblacional, diferencial y territorial, así como sus alternativas de solución.

También es un proceso que demanda un conocimiento sobre la situación desde diferentes perspectivas, contemplar alternativas de solución y la identificación de tensiones entre las personas involucradas, para llegar a acuerdos sobre: las metas que se quieren alcanzar, la manera para lograrlo, la inversión requerida y el tiempo proyectado para generar el cambio. (Decreto 689, 2011)

Es fundamental destacar las formas en que las entidades del Estado colombiano conciben las políticas públicas. El Departamento Nacional de Planeación (DNP) es una entidad eminentemente técnica que impulsa la implantación de una visión estratégica del país en los campos social, económico y ambiental, a través del diseño, la orientación y evaluación de las políticas públicas colombianas, el manejo y asignación de la inversión pública y la concreción de las mismas en planes, programas y proyectos del Gobierno. Los departamentos administrativos son entidades de carácter técnico encargadas de dirigir, coordinar un servicio y otorgar al Gobierno la información adecuada para la toma de decisiones; tienen la misma categoría de los Ministerios, pero sin iniciativa legislativa (Cortes, 2016).

De acuerdo al papel del DNP, es fundamental contar con su perspectiva de la política pública. El seguimiento y la evaluación de las políticas públicas adquiere especial relevancia en la práctica de gobierno:

El cual, inspirado en los modelos del sector privado, parte de definir a los administradores públicos como gerentes responsables de hacer eficiente el gasto y maximizar el valor público de las intervenciones estatales. (Departamento Nacional de Planeación, 2009)

Teniendo en cuentas las visiones presentadas en las líneas anteriores, se puede concluir que las políticas públicas son un mecanismo importante, donde el Gobierno nacional genera un relacionamiento con la población civil, debido a que, a través de la política pública, se plantean soluciones concretas que buscan atender las necesidades 
de la población. La correcta implementación de las mismas puede derivar o no en un eficiente gasto de los recursos públicos y en la atención oportuna, efectiva y eficiente de las necesidades de una Nación.

\section{Entendiendo las dinámicas socioeconómicas de las regiones de Colombia: un estudio de caso en el Pacífico}

Hablar del Pacífico es un buen ejemplo para ilustrar y entender las dinámicas socioeconómicas de Colombia, lo cual es un factor clave en la estructuración y puesta en marcha de políticas públicas eficaces, eficientes y con gran probabilidad de funcionamiento.

El Pacífico colombiano es una región geográfica y natural que abarca los territorios de cuatro departamentos: Chocó, Valle del Cauca, Cauca y Nariño (Romero, 2009). Esta región, con sus $8^{\prime} 410.059$ habitantes, abarca un territorio de $83.170 \mathrm{~km}^{2}$ y equivale al $7 \%$ del territorio nacional.

Mientras que el litoral Pacífico es una subregión geográfica y natural que se encuentra dentro de la región del Pacífico y que abarca todos los municipios del departamento de Chocó, solo Buenaventura en el Valle del Cauca, parte del Cauca y Nariño, donde hay una gran variedad de ecosistemas, especies, fauna y flora endémicas. Esta zona está habitada por más de 1'910.957 personas. A pesar de su riqueza natural y endémica, la economía del litoral es una de las más rezagadas del país; sus indicadores sociales y de bienestar son muestra del enorme rezago relativo al litoral, en comparación con el Pacífico Andino, pese a estar dentro de la misma región.

Para estudiar las brechas socioeconómicas en la región del Pacífico, se analizarán tres indicadores: línea de pobreza o incidencia de Pobreza Monetaria, Índice de Pobreza Multidimensional (IPM) y Necesidades Básicas Insatisfechas (NBI).

El primero se refiere únicamente a la privación monetaria, por lo que se calcula la proporción de personas que se encuentran debajo del mínimo ingreso que se considera necesario para satisfacer necesidades vitales. Sin embargo, este indicador puede ser muy impreciso, dado que el ingreso necesario para cumplir con este objetivo depende de la edad, condiciones de salud, género, ubicación, atmosfera epidemiológica, tradiciones de la familia y enfermedades crónicas. Por ende, un único valor para toda la población colombiana puede no ser adecuado para todas las personas del litoral Pacífico. 
Por consiguiente, para lograr un análisis más directo y acertado, se pueden utilizar otros índices como el NBI y el IPM. El NBI mide la pobreza de la privación de uno de los siguientes indicadores: viviendas sin hacinamiento crítico, condiciones físicas propias para el alojamiento humano, servicios adecuados, baja dependencia económica y niños en edad escolar que asisten a la escuela. No obstante, este índice no tiene en cuenta la privación de buenas condiciones de salud ni de nutrición. Por lo tanto, para esto se analiza el IPM, el cual tiene en cuenta 15 privaciones en las que se incluyen las dimensiones de: educación en el hogar, bienestar de niños y jóvenes, salud, trabajo, vivienda y acceso a servicios públicos.

Si no se cumple con cinco de estas, el hogar se considera pobre. Por ende, se podrían presentar diferencias entre los tres índices si los ingresos mínimos para sobrepasar la línea de pobreza no son suficientes para que una persona cumpla con los requisitos del IPM y NBI, por lo cual es importante hacer un análisis con estas tres medidas. A continuación, se realizará un análisis de estos índices en el litoral Pacífico, en comparación con el Pacífico Andino y el promedio nacional.

\subsection{Pobreza Monetaria y desigualdad}

La pobreza en el litoral Pacífico era de 62,4\% en 1993 y de 64,1\% en 2005, frente a valores de $55,8 \%$ y $51,2 \%$ en los mismos años para el total nacional.

Sin embargo, al observar los datos departamentales, que incluyen el litoral como Chocó, Cauca, Nariño y Valle del Cauca, la tendencia del indicador IPM, desde el 2002 hasta el 2016, sí presenta una tendencia a la baja, pasando de 57,5\% (2002) a 44,7\% (2016), frente a un paso de la incidencia nacional del 55,1\% (2002) al $34,9 \%$ (2016). No obstante, la región Pacífica sigue teniendo niveles de pobreza mucho más altos que el resto del país. Particularmente, en 2015 el Chocó era el departamento con mayor incidencia de la pobreza de la región, seguido por Cauca y Nariño. Este último departamento mejoró significativamente sus indicadores, superando a Cauca y acercándose a la media nacional.

Valle ha presentado valores de pobreza monetaria significativamente más bajos a los de la región y a la media nacional en el periodo estudiado. Esto puede deberse a la presencia de Cali en el departamento, que tiene mejores indicadores que el resto de los municipios de Valle.

En cuanto al Índice de Pobreza Extrema, los valores departamentales de la región pasan del 24,7\% (2002) al 19,9\% (2016) mientras que los nacionales pasan del 21,4\% 
(2002) al 11,6\% (2016). Sin embargo, Valle es el departamento de la región que presenta mejores niveles de pobreza extrema, los cuales fueron de 6,2\%. En cuanto a Chocó y Cauca, las diferencias entre 2002 y 2016 son mínimas, y en el caso de ambos departamentos se está por encima de la media nacional en todos los años.

En cuanto al Gini, el litoral también presenta niveles más altos que el total nacional. De hecho, una gran parte de los indicadores de Gini en 1993 de los municipios del litoral son más altos que la media nacional, o en todo caso no están muy lejos de ella. Más allá, cuando hablamos de los indicadores del año 2005, se encuentra que todos los municipios del litoral Pacífico presentan un indicador de desigualdad más alto que la media nacional. Además, hay varias observaciones que están por encima de ambas medidas.

\subsection{Necesidades Básicas Insatisfechas (NBI)}

Para entender las NBi del Pacífico, también se tiene en cuenta la ciudad de Medellín (Antioquia) y el promedio nacional en el análisis, como se evidencia en el gráfico 1 , el cual hace palpable la gran brecha entre las ciudades capitales del Pacífico Andino

Gráfico 1. NBI litoral vs. Pacífico Andino

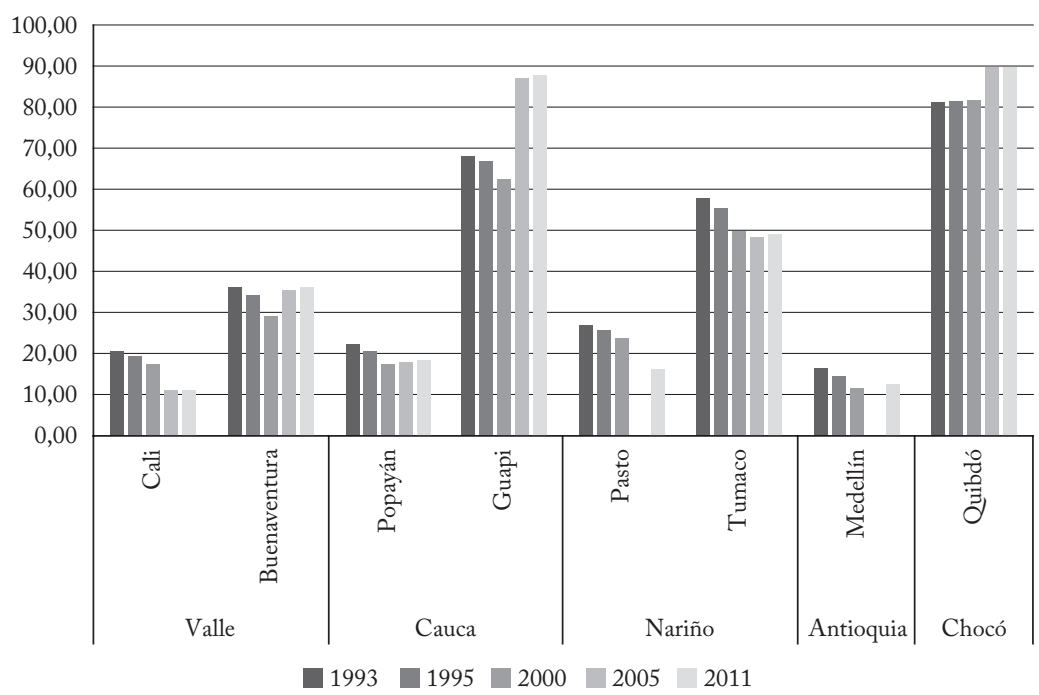

Fuente: elaboración propia a partir de datos de Juan Camilo Cárdenas (2018), publicados en artículo de la Revista Portafolio 
y Antioquia, y las ciudades más representativas del litoral, en cuanto a las NBI desde 1993 hasta el 2011. Mientras la pobreza, medida en término de NBI, cayó en todas las ciudades capitales evaluadas, en las ciudades del litoral las NBI han persistido o han empeorado. Los casos más alarmantes son Guapi y Quibdó, en los que este indicador muestra una disminución en la calidad de vida, de manera contraria a la tendencia nacional. En particular, Quibdó, a pesar de ser una de las 13 ciudades principales del país, no ha tenido mejorías en las condiciones de bienestar de sus habitantes.

Si la tendencia en los indicadores de la calidad de vida de los habitantes de cada uno de estos municipios continúa como hasta ahora, a Buenaventura le faltarían 4.129 años para alcanzar las condiciones de vida de Cali, aunque están tan solo a dos horas y media; y a Tumaco, 138 años para alcanzar las condiciones de vida de Pasto. Mientras que, para Guapi y Quibdó, alcanzar respectivamente a Popayán y a Medellín no sería posible, debido a que empeoraron; el reto de estos municipios es revertir la tendencia que han tenido en los últimos años (Mosquera, 2018).

Este rezago, entre la calidad de vida del litoral Pacífico y el Pacífico Andino, es una alerta para reducir estas brechas entre la periferia y el centro del país. A su vez es una muestra de los alto niveles de desigualdad que enfrenta Colombia, lo cual es necesario tener en cuenta a la hora de diseñar políticas públicas eficientes y efectivas. Bajo este contexto, difícilmente una política diseñada para reducir la pobreza en el departamento del Valle del Cauca genere el mismo efecto en Cali que en Buenaventura, por lo que, de acuerdo con esto, las políticas deben diferenciar las características socioeconómicas dentro de un mismo departamento.

\subsection{Pobreza Multidimensional}

En los indicadores del Índice de Pobreza Multidimensional, el litoral presenta, una vez más, valores más altos a los de la media nacional. Particularmente, para el año 2005 la media nacional indicaba que el 69,5\% de las personas tenían privación en cinco o más indicadores, que pertenecen a las dimensiones de educación, niñez y juventud, vivienda, salud y trabajo. Este valor era del $88 \%$ en el litoral Pacífico.

El litoral Pacífico muestra privaciones más altas que el promedio nacional en 14 de 15 indicadores. Particularmente, los indicadores que muestran mayores niveles de privación en el Pacífico son: empleo formal, con 97,61\% de privación (2005); logro educativo, con 87,40\% (2005); y acceso a agua mejorada, con 67,06\% (2005). 
Luego del anterior análisis de coyuntura económica, en contraste con el Pacífico Andino, en el litoral Pacífico se pueden observar las brechas que existen en departamentos de una misma región del país. Esto nos da una idea del nivel de desigualdad que existe al interior de Colombia. Estos elementos deben ser tenidos en cuenta al formular políticas públicas; para el control y vigilancia fiscal en el país, comprender las realidades locales es vital.

A modo de conclusión, un análisis meramente económico en términos de indicadores de pobreza y desigualdad evidencia las brechas tan abismales que se pueden encontrar en una misma región de Colombia (Barragán, 2016). Estos elementos son clave para considerar la estructuración de políticas públicas, estas circunstancias pueden aportar a la discusión, para tratar de entender las razones por las cuales una política funciona mejor en un departamento u otro, o en un municipio u otro, siendo ambos vecinos (como es el caso de Buenaventura y Cali).

\section{Metodología y descripción de los datos}

\subsection{Datos}

En la presente investigación se consolidó un panel de datos con las siguientes bases:

1. Consolidado de los hallazgos de los reportes de rendición de cuenta, que reportan las Contralorías territoriales al sistema interno de la Auditoría General de la República (2017). Estas bases cuentan con datos desde el 2014 hasta el 2017, con información de los hallazgos y la cuantía de los hallazgos.

2. Índice desempeño fiscal de Terridata del DNP. Esta información está disponible desde el año 2014 hasta el 2017.

3. Índice Transparencia por Colombia. Estos datos muestran información sobre el índice de transparencia de los 32 departamentales y las 32 gobernaciones del país, de 2014 y 2015.

4. Estadísticas del DANE. Se obtuvo información de varias bases de datos de variables como población, PIв per cápita, pobreza extrema y Gini por departamento. En algunas ocasiones se tiene información desde 2014 hasta 2017, y en otras oportunidades se cuenta con menos cantidad de años. 
La unidad de observación de los datos de esta investigación son los departamentos de Colombia.

\subsection{Variables de interés}

Hallazgos: número de hallazgos por departamentos.

Gini: índice que mide la desigualdad en un departamento.

Pobreza extrema: porcentaje de personas en pobreza extrema en un departamento.

Índice de corrupción departamental de Transparencia por Colombia.

Otras variables:

PIB Per Cápita: a nivel de departamentos .

Población: número de habitantes por departamento.

Departamentos: los 32 departamentos de Colombia.

\subsection{Características de los datos}

Para estimar la correlación de hallazgos de corrupción, se usan las siguientes variables: indicadores de desempeño fiscal de los departamentos, índice de pobreza extrema, el índice Gini, pIB per cápita de los departamentos y variables demográficas. Procedemos a realizar inicialmente un ejercicio descriptivo de las variables hallazgo en relación al índice de desempeño fiscal, para los departamentos de Chocó, La Guajira, Cesar, Atlántico y Cundinamarca.

De acuerdo con el gráfico 2, en el caso de Chocó, se nota un incremento en los hallazgos y en el índice de desempeño fiscal de forma simultánea.

Este es un dato importante, el cual lleva a pensar que, de forma descriptiva, habría una correlación entre las variables: el número de hallazgos y el índice de desempeño fiscal de los departamentos.

De acuerdo con el gráfico 3, se observa que, en el caso de Cesar, aunque no tan pronunciado como el caso de Chocó, parece estar sucediendo una situación similar. De forma descriptiva, parece haber una correlación entre el índice de desempeño fiscal y la cantidad de hallazgos, al caer los hallazgos se evidencia una leve caída en el desempeño fiscal del departamento.

En el caso de La Guajira, se evidencia un comportamiento parecido al caso de Cesar, como muestra el gráfico 4. 
Gráfico 2. Hallazgos vs. índice de desempeño fiscal en Chocó

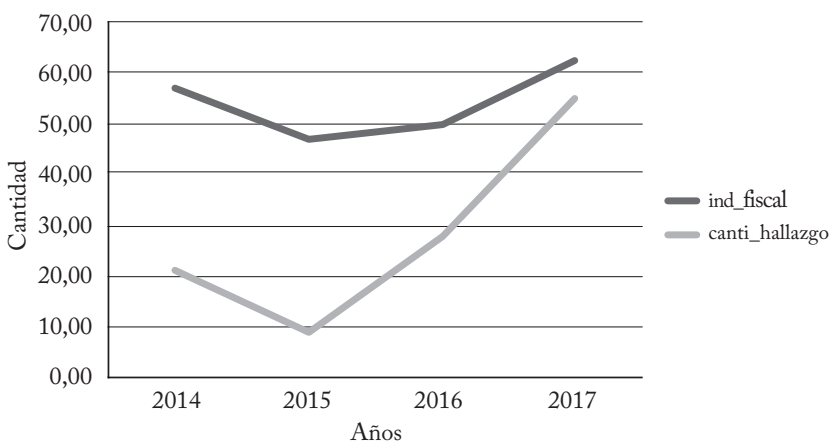

Gráfico 3. Hallazgos vs. índice de desempeño fiscal en Cesar.

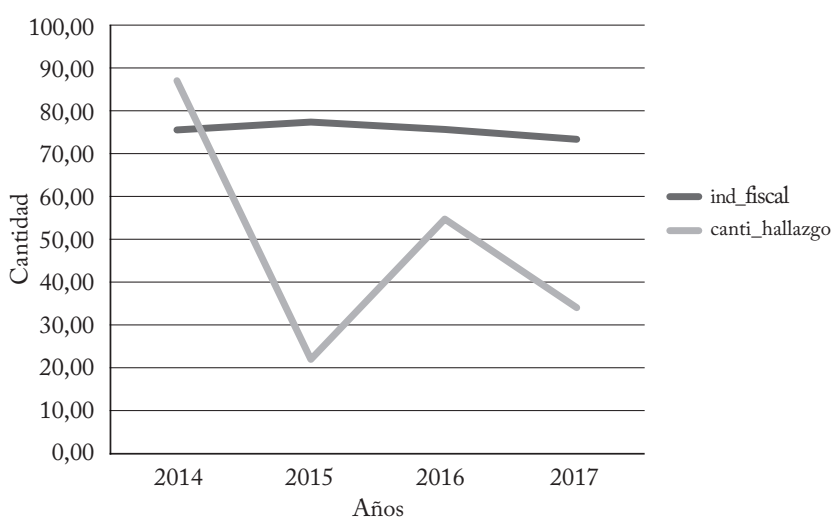

Gráfico 4. Hallazgos vs. índice de desempeño fiscal en La Guajira.

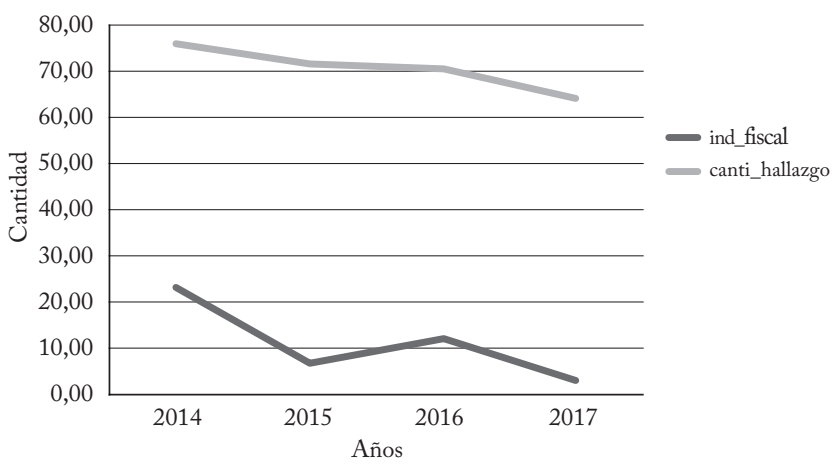


Gráfico 5. Hallazgos vs. índice de desempeño fiscal en Cundinamarca.

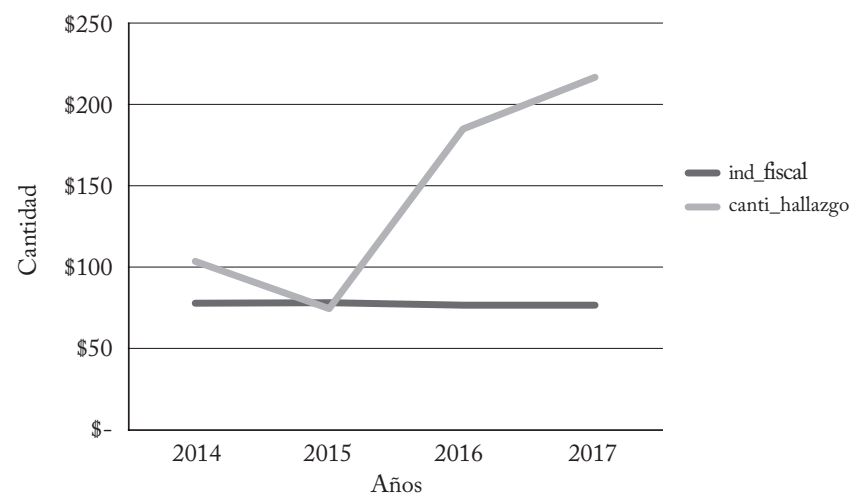

De acuerdo con la muestra anterior, parece que, en los departamentos, cuyo rango de calificación es riesgoso y vulnerable, el comportamiento del índice de desempeño fiscal es parecido al número de hallazgos que se generan. Mientras que, en los casos de departamentos que cuentan con un rango de calificación sostenible en el índice de desempeño fiscal, parece no estar correlacionado el número de hallazgos (como en los casos de Atlántico y Cundinamarca). En el caso de Cundinamarca, el gráfico 5 parece mostrar que no hay relación entre el incremento en los hallazgos y el índice de desempeño fiscal.

Gráfico 6. Descriptiva de las cinco variables de interés para Cesar

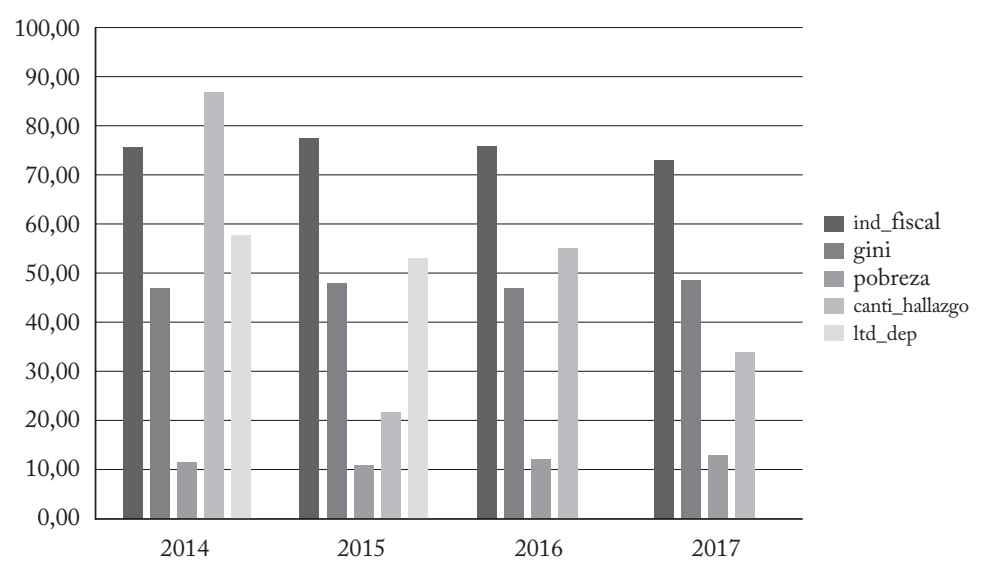


Volviendo a los casos de Chocó y Cesar, revisando los hallazgos vs. las otras variables, de la base de datos en el periodo 2014 a 2017 para mirar la relación entre los hallazgos, el índice de transparencia por Colombia y las otras variables.

De acuerdo con los gráficos 6 y 7, para el caso de Cesar, los hallazgos disminuyeron y el índice departamental de transparencia también disminuyó a lo largo del tiempo, a su vez que aumentó el desempeño fiscal de los departamentos. Este es un dato importante para analizar y estudiar más a fondo. Otro dato importante, de forma descriptiva, que vale la pena resaltar, es que, a medida que disminuye la desigualdad, disminuye levemente la pobreza.

Luego de hacer un análisis descriptivo de las variables, se encontró que, en algunos departamentos con altas tasas de pobreza extrema, como Chocó, Cesar y La Guajira, podría existir una correlación entre el índice de desempeño fiscal y la cantidad de hallazgos. En el caso de Chocó, se nota un incremento en los hallazgos y en el índice de empeño fiscal de forma simultánea, como muestra el gráfico.

La hipótesis principal de este estudio plantea que los departamentos más rezagados, en términos socioeconómicos, presentan mayor número de hallazgos y dinero perdido a raíz de corrupción. A modo de especulación, se plantean los siguientes escenarios:

- Los departamentos con mayor desigualdad (Gini) cuentan con menor número de hallazgos de hechos de corrupción.

- En cuanto incrementa el número de hallazgos por corrupción, los departamentos mejoran el índice de desempeño fiscal.

Gráfico 7. Descriptiva de las cinco variables de interés para Chocó

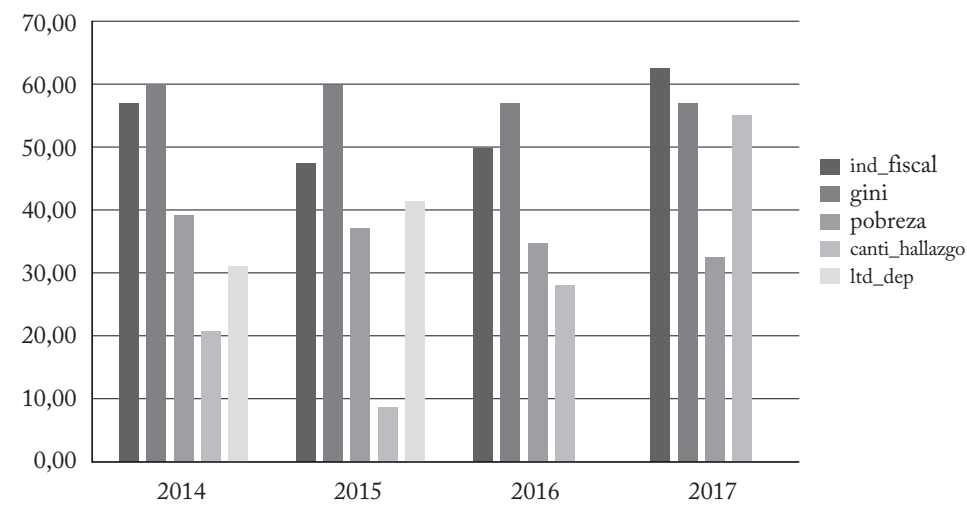


- Los ingresos de los habitantes de un departamento, medidos en términos de PIB per cápita, podrían estar correlacionados con el número de hallazgos de corrupción que realizan las contralorías territoriales.

- Los departamentos más rezagados, que menos reportan hallazgos de corrupción, podrían contar con niveles de corrupción más altos.

\subsection{Metodología}

Se plantea la estimación de regresiones lineales con efectos fijos, aplicando la metodología de panel y usando la variable hallazgos de corrupción como variable respuesta.

Dado que se cuenta con datos panel del periodo de 2014 hasta 2017 en todas las variables, excepto el índice de transparencia a nivel departamental con sólo dos periodos (2014 y 2015), la estimación de regresiones lineales por efectos fijos busca controlar por otras variables que no cuenta el panel. Esto permite tener unos resultados mucho más robustos y de mayor credibilidad. Para esto, se correrán cinco regresiones, para ver si existe correlación entre los hallazgos y otras variables observables del panel. Adicionalmente, esta información cuantitativa se complementará con el análisis de información cualitativa, que permita comprender mejor este estudio y brindar información precisa para la toma de decisiones y recomendaciones de políticas públicas.

$$
Y_{i t}=\beta_{0}+\beta_{1} X_{i t}+\mu_{i t}(1)
$$

$Y_{i}=$ número de hallazgos por departamento

El subíndice $i=$ hace referencia a los departamentos

El subíndice t: hace referencia al tiempo en años (2014 a 2017)

$\beta_{0}=$ término constante del regresor (Intercepto)

$\beta_{1} X_{i t}:=$ vector índice Gini

$\mu_{i t}=$ vector de errores o variables omitidas

Así mismo, se hacen 4 estimaciones más, bajo el mismo modelo, incluyendo las variables: índice desempeño fiscal, logaritmo natural del PIв per cápita, índice de trasparencia departamental e índice de pobreza extrema por departamento. 


\subsection{Limitaciones de la metodología}

Es importante resaltar que, para realizar este tipo de estimaciones, siempre es preferible contar con un panel del dato de muchas observaciones, dado que se cuenta con datos a nivel departamental, pues no fue posible construir una base de datos a nivel municipal; tan solo se cuenta con 128 observaciones, en el caso del índice de transparencia departamental, solo se cuenta con 64 observaciones. El poco número de observaciones podría limitar el modelo en cuento a la varianza, lo cual no permitiría mostrar significancia en los betas.

\section{Resultados estimación estrategia empírica}

Luego de hacer las estimaciones a los 5 modelos econométricos, se encuentra significancia al 10\% entre la variable hallazgo y las variables Gini y PIB per cápita, lo cual indica que hay correlación entre esas variables. A continuación, se exponen los resultados del ejercicio propuesto (tabla 1 ).

Luego de correr la regresión entre la variable hallazgos y las variables pobreza extrema, índice de desempeño fiscal e índice de transparencia por Colombia,

Tabla 1. Estimación lineal por efectos fijos del número de hallazgos por departamento ${ }^{3}$

\begin{tabular}{|l|c|}
\hline \multicolumn{1}{|c|}{ Variables } & $\begin{array}{c}\text { Número de Hallazgos } \\
\text { por departamento }\end{array}$ \\
\hline Gini & $0,02301^{*}$ \\
& $(4,6572)$ \\
\hline Índice desempeño fiscal & 0,1674 \\
& $(17,366)$ \\
\hline (3) Logaritmo natural del PIB per cápita & $0.04662^{*}$ \\
& $(59,493)$ \\
\hline (4) Índice de trasparencia departamental & 0,9852 \\
& 0.833586 \\
\hline (5) Índice de pobreza extrema por departamento & 0,9028 \\
& $(3,89388)$ \\
\hline
\end{tabular}

*** $\mathrm{p}<0.01,{ }^{* *} \mathrm{p}<0.05,{ }^{*} \mathrm{p}<0.1$

Fuente: cálculos del autor

3 Número de hallazgos por departamento: Errores estándar en paréntesis. ${ }^{* * *} \mathrm{p}<0.01,{ }^{* *} \mathrm{p}<0.05,{ }^{*}$ $\mathrm{p}<0.1$

Las tres estrellas indican que es significativo al 1\%, las dos estrellas indican que es significativo al $5 \%$ y una estrella indica que es significativo al 10\%. 
se muestra que los coeficientes no son estadísticamente significativos, lo que nos permite afirmar que en esta instancia no hay correlación entre las variables.

La regresión del modelo (1) arroja significancia conjunta con un F de 5.39912, que permite rechazar la hipótesis nula de que los coeficientes sean iguales a cero. Por otra parte, la variable Gini arroja significancia individual al $90 \%$ de confianza. En cuanto a la interpretación de los coeficientes, podemos afirmar que, ante el incremento de $1 \mathrm{pp}$ en el Gini, genera un efecto promedio sobre la variación de los hallazgos de 0.02301 unidades.

Los resultados de la regresión del modelo (2) no arrojan significancia conjunta en el F de 1.93637, lo cual no permite rechazar la hipótesis nula de que los coeficientes sean iguales a cero. Por otra parte, la variable índice de desempeño fiscal por departamento no es significativa. Esto nos permite afirmar que no existe correlación entre la variable hallazgos y el índice de desempeño fiscal. Sin embargo, es importante seguir explorando mecanismos que permitan testear si hay correlación entre ambas variables con otro tipo de metodología, dado que, como lo vimos en numerales anteriores, gráficamente sí existía correlación, por lo menos de forma descriptiva.

Los resultados de la regresión del modelo (3) arrojan significancia conjunta con un F de 4.07053, que permite rechazar la hipótesis nula de que los coeficientes sean iguales a cero. Por otra parte, la variable logaritmo natural del PIв per cápita arroja significancia individual al 90\% de confianza. En cuanto a la interpretación de los coeficientes, podemos afirmar que, ante el incremento de $1 \%$ en el PIв per cápita, genera un efecto sobre la variación de los hallazgos de 0.04662 unidades.

Los resultados de la regresión del modelo (4) no arrojan significancia conjunta en el F de 0.000348 , lo cual no permite rechazar la hipótesis nula de que los coeficientes sean iguales a cero. Por otra parte, el índice de trasparencia departamental no es significativa. Esto permite afirmar que no existe correlación entre la variable hallazgos y el índice de trasparencia departamental. Sin embargo, hay que mencionar que esta variable solo tiene dos periodos de tiempo, por lo que no se cuenta con muchas observaciones, lo que hace que no haya mucha variabilidad para encontrar significancia conjunta. Sería interesante testear esta variable cuando surjan más datos, y así poder hacer una estimación más sólida.

Los resultados de la regresión del modelo (5) no arrojan significancia conjunta en el F de 0.01502, lo cual no permite rechazar la hipótesis nula de que los coeficientes sean iguales a cero. Por otra parte, el índice de pobreza extrema por departamento 
no es significativo. Esto permite afirmar que no existe correlación entre la variable hallazgos y el índice de pobreza extrema por departamento.

\section{Conclusiones}

Teniendo en cuenta los resultados obtenido en el presente artículo, a modo de conclusiones, se resaltan los siguientes aspectos:

Las políticas públicas no funcionan de la misma manera en todo el territorio nacional. De acuerdo con las características sociales, culturales y económicas, se deberían priorizar los programas y proyectos.

Se puede evidenciar la importancia de comprender las dinámicas regionales de Colombia, para la implementación y puesta en marcha de políticas públicas, efectivas, eficaces y eficientes, que permitan cerrar brechas en la sociedad.

La desigualdad podría estar profundizando el problema de la corrupción en Colombia. Los departamentos con mayor debilidad fiscal, y mayor desigualdad, podrían estar presentando mayores casos de hallazgos de corrupción y detrimento patrimonial. Por esto, para estructurar políticas públicas de control y vigilancia fiscal, no solo basta con enfocarse en reformar las Contralorías territoriales per se, sino que se deben pensar también programas que permitan generar un fortalecimiento social y económico a las regiones más rezagadas del país. Se trata entonces de pensar una política pública integral, que abarque otros escenarios de la sociedad.

Finalmente, y como se ha reiterado a lo largo del presente documento, se hace un llamado al Gobierno nacional, y a todos los órganos e instancias involucradas, a desarrollar y pensar políticas públicas que permitan cerrar brechas, teniendo en cuenta aspectos basados en la realidad de los territorios colombianos, como la desigualdad, la debilidad institucional, los índices de corrupción, y las características sociales y económicas. A su vez, se requiere fortalecer paralelamente las instituciones, con el propósito de que estas tengan un mejor desempeño.

\section{Referencias}

Alcaldía Distrital de Bogotá D. C. (2017). Guía para la formulación e implementación de políticas públicas del Distrito. [Decreto 689 de 2011].

Auditoría General de la República. (2017). Base de datos. Recuperado de http://www. auditoria.gov.co/ 
Barragán, D. (2016). La construcción de la mentalidad democrática como necesidad en el posconflicto. Revista Via Inveniendi et Iudicandi, 11(1), 37-57.

Benavente, M. C. (2014). Políticas públicas para la igualdad de género. Santiago de Chile, Chile: Cepal.

Blanco C (2015) la conexión entre descentralización territorial colombiana e integración andina en Revista Análisis Politico, Volumen 28, Número 84, 23-56.

Cárdenas, J. C. (2018). Desarrollo con, desde y para el Pacífico. Portafolio. Recuperado de https://www.portafolio.co/opinion/juan-camilo-cardenas/ desarrollo-con-desde-y-para-el-pacifico-analisis-516746

Cortes S (2016) Derechos humanos en las políticas de paz y posconflicto en Colombia en Revista Via Inveniendi et Iudicandi, Vol. 11, N. 1 / enero-junio 2016, pp. 129-145.

Procuraduría General de la Nación [PGN]. (2011). Metodología para la actualización preventiva de la Procuraduría General de la Nación en las diferentes etapas del ciclo de las políticas públicas. Bogotá D.C: Programa de Modernización.

Departamento Nacional de Planeación [DNP]. (2009). Guia para el seguimiento de políticas públicas. Bogotá.

Constitución política de Colombia [Const.]. (1991).

Departamento Administrativo Nacional de Estadística [DANE]. (17 de mayo de 2019). Estadísticas por tema. Recuperado de http://www.dane.gov.co/

Departamento Nacional de Planeación [DNP]. (25 de julio de 2014). Funciones DNP. Recuperado de https://www.dnp.gov.co/DNPN/la-entidad/funciones-dnp

Mosquera, L. (2018). Los retos socioeconómicos del presidente Iván Duque con el litoral Pacífico. Semana. Recuperado de https://www.semana.com/opinion/articulo/ los-retos-socioeconomicos-del-presidente-ivan-duque-con-el-litoral-pacifico/582940

Parsons, W. (1995). Public Policy: An Introduction to the Theory and Practice of Policy. Aldershot, Reino Unido: Brookfield, 247-250.

Romero, J. (2009). Geografía económica. Cartagena, Colombia: Banco de la República.

Sánchez, J. A. (2012). Biodiversidad, conversación y desarrollo. Bogotá, Colombia: Universidad de los Andes.

Torres, B. (2006). Metodología de la investigación para administración, economia, humanidades $y$ ciencias sociales. México, Pearson Educación. 